TITLE:

\title{
Chain contraction and nonlinear stress damping in primitive chain network simulations
}

\section{AUTHOR(S):}

Furuichi, Kenji; Nonomura, Chisato; Masubuchi, Yuichi; Watanabe, Hiroshi

\section{CITATION:}

Furuichi, Kenji ... [et al]. Chain contraction and nonlinear stress damping in primitive chain network simulations. JOURNAL OF CHEMICAL PHYSICS 2010, 133(17): 174902.

\section{ISSUE DATE:}

2010-11

\section{URL:}

http://hdl.handle.net/2433/147216

\section{RIGHT:}

Copyright 2010 American Institute of Physics. This article may be downloaded for personal use only. Any other use requires prior permission of the author and the American Institute of Physics. The following article appeared in JOURNAL OF CHEMICAL PHYSICS133, 174902 (2010) and may be found at 


\title{
Chain contraction and nonlinear stress damping in primitive chain network simulations
}

\author{
Kenji Furuichi, ${ }^{1}$ Chisato Nonomura, ${ }^{1}$ Yuichi Masubuchi, ${ }^{2, a)}$ and Hiroshi Watanabe ${ }^{2}$ \\ ${ }^{1}$ TOYOBO Co., Ltd., Otsu, Shiga 520-0292, Japan \\ ${ }^{2}$ Institute for Chemical Research, Kyoto University, Uji, Kyoto 611-0011, Japan
}

(Received 14 June 2010; accepted 24 September 2010; published online 1 November 2010)

\begin{abstract}
Doi and Edwards (DE) proposed that the relaxation of entangled linear polymers under large deformation occurs in two steps: the fast chain contraction (via the longitudinal Rouse mode of the chain backbone) and the slow orientational relaxation (due to reptation). The DE model assumes these relaxation processes to be independent and decoupled. However, this decoupling is invalid for a generalized convective constraint release (CCR) mechanism that releases the entanglement on every occasion of the contraction of surrounding chains. Indeed, the decoupling does not occur in the sliplink models where the entanglement is represented by the binary interaction (hooking) of chains. Thus, we conducted primitive chain network simulations based on a multichain sliplink model to investigate the chain contraction under step shear. The simulation quantitatively reproduced experimental features of the nonlinear relaxation modulus $G(t, \gamma)$. Namely, $G(t, \gamma)$ was cast in the time-strain separable form, $G(t, \gamma)=h(\gamma) G(t)$ with $h(\gamma)=$ damping function and $G(t)$ $=$ linear modulus, but this rigorous separability was valid only at times $t$ comparable to the terminal relaxation time, although a deviation from this form was rather small (within $\pm 10 \%$ ) at $t>\tau_{R}$ (longest Rouse relaxation time). A molecular origin of this delicate failure of time-strain separability at $t \sim \tau_{R}$ was examined for the chain contour length, subchain length, and subchain stretch. These quantities were found to relax in three steps, the fast, intermediate, and terminal steps, governed by the local force balance between the subchains, the longitudinal Rouse relaxation, and the reptation, respectively. The contributions of the terminal reptative mode to the chain length relaxation as well as the subchain length/stretch relaxation, not considered in the original DE model, emerged because the sliplinks (entanglement) were removed via the generalized CCR mechanism explained above and the reformation of the sliplinks was slow at around the chain center compared to the more rapidly fluctuating chain end. The number of monomers in the subchain were kept larger at the chain center than at the chain end because of the slow entanglement reformation at the center, thereby reducing the tension of the stretched subchain at the chain center compared to the DE prediction. This reduction of the tension at the chain center prevented completion of the length equilibration of subchains at $t \sim \tau_{R}$ (which contradicts to the DE prediction), and it forces the equilibration to complete through the reptative mode at $t \gg \tau_{R}$. The delicate failure of time-strain separability seen for $G(t, \gamma)$ at $t \sim \tau_{R}$ reflects this retarded length equilibration. (C) 2010 American Institute of Physics. [doi:10.1063/1.3502681]
\end{abstract}

\section{INTRODUCTION}

It has been widely accepted that the entangled polymer dynamics can be described by the models where the effect of entanglement is represented as a geometrical constraint on the chain dynamics. The most successful model is the tube model where the constraint is represented by a tube surrounding the chain and the chain diffuses along this tube. It has been believed that the tube model captures the basic features of polymer dynamics at equilibrium ${ }^{1}$ as well as under large deformation/fast flow in the nonlinear viscoelastic regime. $^{2}$ Indeed, the Doi-Edwards (DE) model describes quantitatively the magnitude of nonlinear stress damping of entangled linear chains under large deformation.

The DE model considers that the nonlinear relaxation

\footnotetext{
a) Author to whom correspondence should be addressed. Electronic mail: mas@scl.kyoto-u.ac.jp.
}

under step-shear deformation proceeds in two steps. In the first step, the affinely deformed/stretched polymer chain is assumed to contract along the tube to recover its equilibrium length. This contraction occurs with the longitudinal Rouse mode having the characteristic time $\tau_{R} \propto M^{2}$ ( $M=$ chain molecular weight). On the contraction, the chain abandons a portion of the tube, and the modulus exhibits a nonlinear decrease according to the magnitude of applied strain. After the contraction, the orientational relaxation is considered to occur as the second step through reptation (curvilinear diffusion) of the chain along the tube. This reptation process is identical to that under small strains and thus the terminal relaxation time under large strains agrees with that in the linear viscoelastic regime.

This two-step relaxation behavior has been experimentally observed, ${ }^{3}$ and the relaxation modulus in the second step (at times $t$ longer than a certain characteristic time $\tau_{k}$ ) 
can be expressed in a time-strain separated (factorized) form, $G(t, \gamma)=h(\gamma) G(t)$ with $h(\gamma)$ and $G(t)$ being the damping function attributable to the chain contraction and the linear relaxation modulus reflecting the orientational relaxation, respectively. ${ }^{4}$ The damping function deduced from the DE model, $h^{\mathrm{DE}}(\gamma)$, represents a decrease of a number of the entanglement segment per chain (due to the chain contraction) and an increase of the monomer number per this segment (that reduces the tension along the chain backbone). This $h^{\mathrm{DE}}(\gamma)$, calculated in a purely geometrical way without any adjustable parameter, agrees with the $h(\gamma)$ data considerably well. The characteristic time $\tau_{k}$ was reported to be larger than $\tau_{R}$ but proportional to $M^{2}$ (as is the case also for $\tau_{R}$ ). ${ }^{3,4}$

On the other hand, recent experiments suggested that the chain contraction and orientational relaxation processes are coupled with each other and thus the simple DE picture explained above needs to be modified. For example, SanchezReyes and Archer ${ }^{5}$ measured the stress relaxation of polystyrene solutions and found that the time-strain separability of $G(t, \gamma)$ does not accurately hold up to a time considerably larger than the aforementioned $\tau_{k}$. The critical time for timestrain separability, $\tau_{k}{ }^{\prime}$, was reported to be close to the terminal relaxation time, $\tau_{d}$ (rather than $\tau_{R}$ ). Inoue et $a .^{6}$ also measured stress relaxation of polystyrene solutions and reported that the relaxation modulus at $t>\tau_{k}$ involves a secondary, weak relaxation with a characteristic time much longer than $\tau_{R}$.

A molecular picture that may rationalize the coupling of the contraction and orientational relaxation has been proposed in relation to the tube deformation in a direction lateral to the chain backbone. Mhetar and Archer ${ }^{7}$ proposed a partial strand extension model where the chain does not fully retract to the equilibrium length at $t \sim \tau_{R}$ because of the lateral squeezing due to the tube. They considered that this squeezing effect vanishes and the chain recovers its equilibrium length only after the chain escapes the deformed tube and thus the time-strain separability becomes valid only at $t \geq \tau_{d}\left(\gg \tau_{R}\right)$. A similar squeezing effect was pointed out earlier by Marrucci and de Cindio. ${ }^{8}$

Another idea rationalizing the coupling of the chain contraction and orientational relaxation can be found in relation to the convective constraint release (CCR) mechanism. ${ }^{9}$ The CCR mechanism originally proposed by Marrucci ${ }^{9}$ releases the entanglements for a given chain on convection/ contraction of the surrounding chains under flow, but the same type of release should occur on contraction of the surrounding chains under step strain. With this generalized CCR hereafter simply referred to as CCR unless some confusion arises, the contraction under step strain releases the entanglements thereby possibly affecting the orientational relaxation process. This CCR effect can be easily examined with the sliplink model that represents the entanglement as a sliplink between two chains. A sliplink near a chain end vanishes on the chain contraction and a partner chain having entangled through this sliplink exhibits a partial, orientational relaxation due to CCR, which demonstrates coupling between the chain contraction and orientational relaxation.

Chen et al. ${ }^{10}$ simulated the stress relaxation with a single chain sliplink model proposed by Hua and Schieber. ${ }^{11}$ In spite of the coupling between the chain contraction and orientational relaxation incorporated in their model, the simulated results systematically deviated from experimental data with increasing time from $\tau_{R}$ to $\tau_{d}$. They suggested that nonaffine deformation due to force balance around entanglement, being not considered in their mean-field single-chain simulation, may improve the prediction. Indeed, a multichain sliplink model (primitive chain network model ${ }^{12}$ ) incorporating this force balance reproduces the nonlinear stress relaxation modulus quantitatively ${ }^{13}$ and demonstrated the nonaffine deformation of the chains subjected to the step strain due to this force balance (that is in accord to the three-chain theory proposed by Marrucci et al. ${ }^{14}$ ). However, the chain contraction itself and the coupling between the contraction and orientational relaxation were not fully examined.

Thus, we investigated the stress relaxation and chain contraction in the sliplink network through the primitive chain network (PCN) simulations ${ }^{12}$ under step shear. It turned out that the (generalized) CCR mechanism reduces the chain tension at around the chain center compared to the tension deduced from the DE model. This tension reduction leads to the retarded chain contraction (coupled with the orientational relaxation) and the limited validity of rigorous time-strain separability (at $t \geq \tau_{d}$ ). This paper presents details of these results and discusses mechanisms leading to the retarded contraction.

Before closing this section, it should be emphasized that the above discussion intrinsically assumes homogeneity of the strain field. Another possible mechanism for the nonlinear stress damping has been proposed in relation to macroscopic banding or rupture observed for highly entangled polymer solutions and melts with particle tracking velocimetry. ${ }^{15-17}$ According to the observation, the two-step relaxation of highly entangled polymers is due to the macroscopic yielding of specimen rather than the microscopic chain dynamics. It has been also pointed out that some literature data of the nonlinear relaxation modulus could have been obtained under inhomogeneous strain field.

Since PCN is a multichain model where the deformation field is determined by force balance among the chains, the homogeneity of the strain field can be monitored, in principle, with the PCN simulation in a cell of macroscopic size. However, the present study focused on the microscopic chain dynamics and thus conducted the PCN simulation in a microscopic cell containing $\sim 2000$ chains. The strain field, determined by the chains in our cell, exhibited no heterogeneity, which may be partly attributed to the cell size and also to the entanglement density: The simulated chain had $\sim 20$ entanglements and was much less entangled compared to the actual chains ${ }^{15-17}$ exhibiting the shear banding/yielding. Thus, the current study positions itself as a detailed test of the DE assumption of the independence of the contraction and orientation relaxation. The shear banding/yielding might be examined through the PCN simulation for highly entangled chains in a macroscopic cell, but this examination is beyond the scope of the current study and postponed to our future work. 


\section{MODEL AND SIMULATIONS}

The model and code are identical to those used in Refs. 13,18 , and 19 and only briefly described here. In the PCN model, entangled polymers are replaced by a threedimensional network composed of sliplinks (network node) and subchains (strands). Each polymer chain is divided into a sequence of subchains, and each sliplink binds two chains (four subchains) under an assumption that the entanglement is a binary hooking event. The polymer dynamics is fully described through the dynamics of sliplink according to the force balance, the monomer transport between subchains through the sliplink (i.e., chain sliding), and the creation and destruction of the sliplinks due to the constraint renewal at chain ends. Thus the state valuables are the position vector of the sliplinks, $\{\mathbf{R}\}$, the number of monomers in each subchain, $\{n\}$, and the number of subchains in each chain $\{Z\}$. The governing equations were cast in a dimensionless form shown below with unit of length $a=\sqrt{n_{0}} b$, unit of energy $k T$, and unit of time $\tau_{0}=\mathrm{s} a^{2} / 6 k T$ with $b, n_{0}$, and $\mathrm{s}$ being the step length of monomer, the mean equilibrium value of monomer number in the subchain, and the friction coefficient for the sliplink, respectively.

The equation of motion for $\{\mathbf{R}\}$ is given by

$$
\dot{\mathbf{R}}=\frac{1}{2} \sum_{j}^{4} \frac{\mathbf{r}_{j}}{n_{j}}-\frac{1}{3} \nabla \mu+\mathbf{F} .
$$

The first term in the right-hand side represents the force balance among the four subchains bound by the sliplink, and $\mathbf{r}$ is the end-to-end vector of subchain. The second term is the repulsive force arising from the gradient of a chemical potential $\mu$. The free energy $A$, giving $\mu$ as its derivative, is defined by

$$
A= \begin{cases}\varepsilon\left(\frac{\phi}{\langle\phi\rangle}-1\right)^{2} & \text { for } \phi>\langle\phi\rangle \\ 0 & \text { for } \phi \leq\langle\phi\rangle,\end{cases}
$$

where $\phi$ is the local subchain density and $\langle\phi\rangle$ is its average in the whole system. The numerical parameter $\varepsilon$ was fixed at 0.5 , a value reproducing the linear and nonlinear viscoelastic data. ${ }^{13,18}$ The third term in Eq. (1) is a Gaussian random noise characterized by the averages, $\langle\mathbf{F}\rangle=0$ and $\left\langle\mathbf{F}(t) \mathbf{F}\left(t^{\prime}\right)\right\rangle$ $=\delta\left(t-t^{\prime}\right) \mathbf{I}$ with $\mathbf{I}=$ unit tensor.

The kinetic equation for $\{n\}$ is written as

$$
2\left(\frac{n_{i}}{r_{i}}+\frac{n_{i-1}}{r_{i-1}}\right)^{-1} \dot{n}_{i}=\left(\frac{r_{i}}{n_{i}}-\frac{r_{i-1}}{n_{i-1}}\right)-\frac{1}{3} \nabla \mu+f
$$

The left-hand side indicates the rate of change of the number of monomers in the $i$ th subchain, $\dot{n}_{i}$, being transported from the $i-1$ th subchain, multiplied by the reciprocal of the linear density of the monomer averaged over the two adjacent subchains. The first term in the right-hand side represents the tension difference between the adjacent subchains, and the second term, the gradient of chemical potential along the
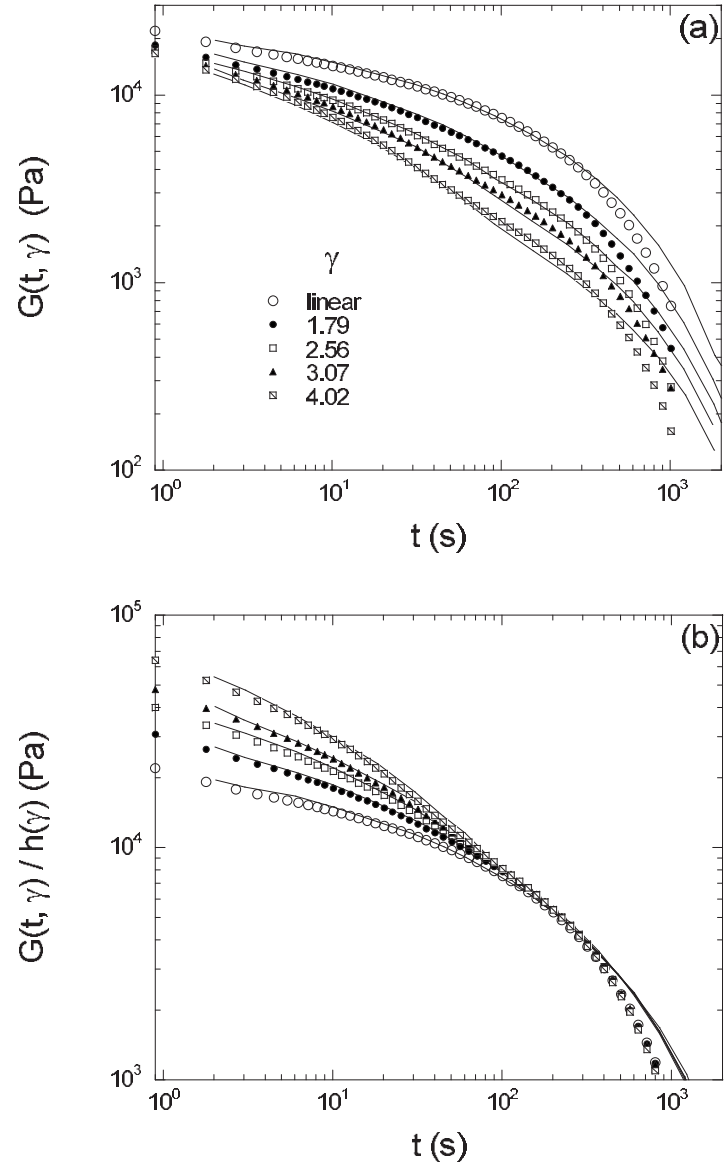

FIG. 1. Comparison of simulation results and the experimental data for PS solution (Ref. 3). (a) Relaxation modulus for various strains; (b) test of time-strain separability. Symbols and solid curves indicate simulations and experimental data, respectively.

chain. The last term is a one-dimensional Gaussian random force characterized by the averages, $\langle f\rangle=0$ and $\left\langle f(t) f\left(t^{\prime}\right)\right\rangle$ $=2 \delta\left(t-t^{\prime}\right) / 3$.

The creation and destruction of the sliplinks, inducing a fluctuation of $\{Z\}$, are triggered by the monomer number at the chain ends. If the monomer number $n$ in the end subchain becomes smaller than a given minimum threshold $(=0.5)$, the sliplink neighboring to the examined subchain is removed. On the other hand, if $n$ exceeds a given maximum threshold $(=1.5)$, a new sliplink is created on the examined subchain by random hooking with a randomly chosen subchain among the surroundings. No creation/destruction of the sliplink is made for $n$ in a range of $0.5<n<1.5$.

Simulations were performed with periodic shear boundary using the Lees-Edwards method in a box of size $16 a^{3}$. The mean segment number density was set to $\langle\phi\rangle=10 / a^{3}$. The system was affinely step-sheared at $t=+0$, and the relaxing stress was calculated as the average over 50 independent runs. The linear relaxation modulus $G(t)$ was calculated from autocorrelation of a microscopic shear stress (microscopic momentum transfer) at equilibrium (under no strain). We evaluated unit modulus $G_{0}$ and unit time $\tau_{0}$ in the simulation by fitting the $G(t)$ data with the simulation, as shown later in Fig. 1. The nonlinear relaxation modulus was evaluated from the stress tensor written as (in the dimensionless form) 


\section{2-4}

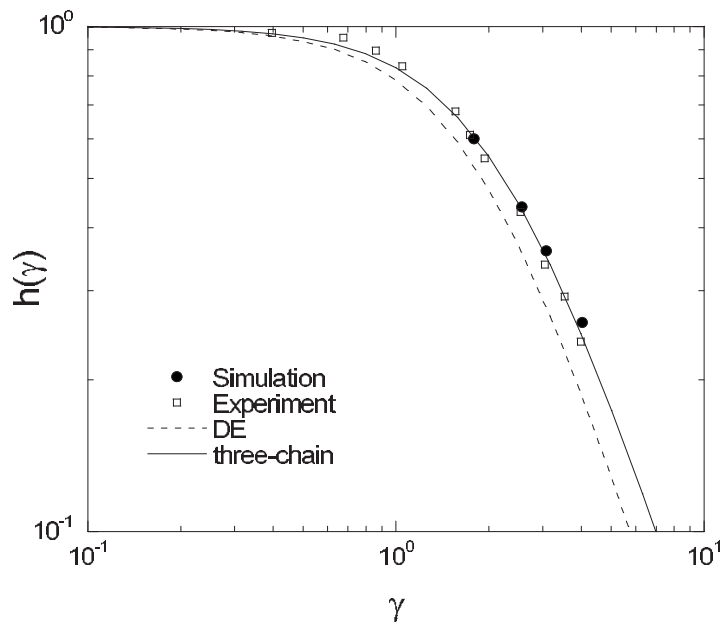

FIG. 2. Damping function obtained from the simulation (filled circle), experiments (open square) (Ref. 3), the three-chain theory (Refs. 14 and 19) (solid curve), and the DE theory without independent alignment approximation (Ref. 2) (dashed curve).

$$
\boldsymbol{\sigma}=\frac{3}{N_{\mathrm{eq}}} \sum \frac{\mathbf{r r}}{n} .
$$

Here, $N_{\text {eq }}$ is the total number of subchains under equilibrium. We did not explicitly take account the osmotic stress [corresponding to the second term in the right-hand side of Eqs. (1) and (3)] since it was rather small compared to the stress given by Eq. (4).

\section{RESULTS}

\section{A. Overview}

Figure 1(a) compares the data of the nonlinear relaxation modulus $G(t, \gamma)$ of a polystyrene (PS)/arclor solution published by Osaki et $a .^{3}{ }^{3}$ (curves) with the $G(t, \gamma)$ of the PCN model (symbols) calculated for the parameters $G_{0}$ $=34000 \mathrm{~Pa}, M_{0}=29700 \mathrm{~g} / \mathrm{mol}$, and $\tau_{0}=0.9 \mathrm{~s}$. The parameters $G_{0}$ and $M_{0}$ are related to each other as

$$
G_{0}=\frac{c R T}{M_{0}},
$$

where $c\left(=0.4 \mathrm{~g} \mathrm{~cm}^{-3}\right)$ is the polymer concentration and $R$ is the gas constant. From $M_{0}$, the average number of $Z$ at equilibrium is obtained as $\langle Z\rangle_{\mathrm{eq}}=M_{w} / M_{0}=23$, where $M_{w} \quad(=6.7$ $\left.\times 10^{5}\right)$ is the molecular weight of the PS sample.

As seen in Fig. 1(a), the simulated $G(t, \gamma)$ agrees quantitatively with the data except at long times $t>300 \mathrm{~s}$ (where the polydispersity of the PS sample needs to be considered to reproduce the data). Figure 1(b) examines the time-strain separability for the measured and simulated $G(t, \gamma)$. The $G(t, \gamma)$ curves for different $\gamma$ values are excellently superposed when reduced by an appropriate damping function $h(\gamma)$, which confirms the time-strain separability at long $t$. Figure 2 shows the $\gamma$ dependence of $h(\gamma)$ giving this superposition. The simulated $h(\gamma)$ agrees with the $h(\gamma)$ data, and its $\gamma$ dependence is slightly weaker than the DE prediction and close to the prediction of the three-chain theory, ${ }^{14}$ as reported earlier. ${ }^{19}$

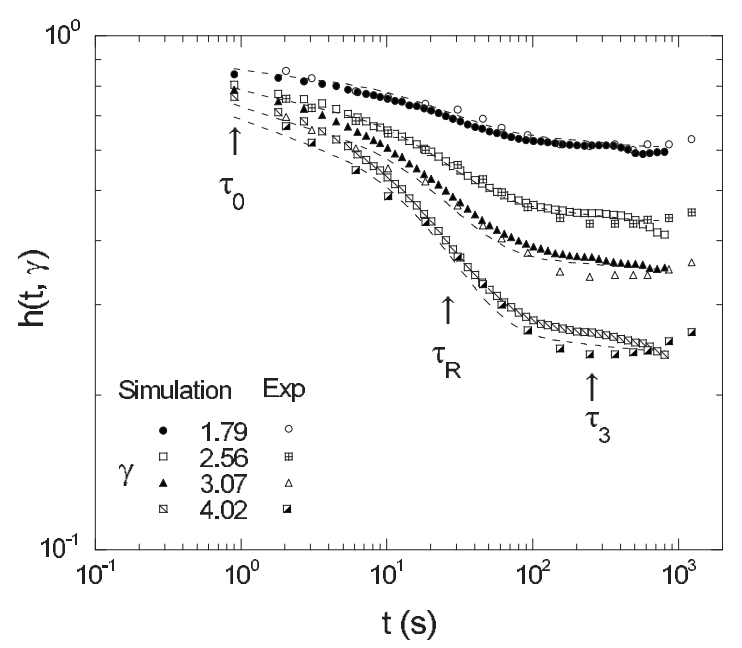

FIG. 3. Time-dependent damping functions obtained from simulations and the experiments (Ref. 3). Dashed lines shows the behavior deduced from Eq. (7). Arrows indicate the unit time $\tau_{0}$, Rouse time $\tau_{R}$ and the longest relaxation time $\tau_{3}$ defined in Eq. (7).

\section{B. Detailed feature of time-strain separability}

The time-strain separability seen in Fig. 2 can be further characterized by a time-dependent damping function

$$
h(t, \gamma) \equiv \frac{G(t, \gamma)}{G(t)}
$$

The experimentally obtained and simulated $h(t, \gamma)$ are shown in Fig. 3 (symbols).

For analysis of $h(t, \gamma)$, we may cast it in an approximate but rather general form proposed by Takahashi et al. ${ }^{20,21}$

$$
h(t, \gamma)=h(\gamma)[1+\{\alpha(\gamma)-1\} f(t, \gamma)]^{2}
$$

Here, $\alpha(\gamma)$ is the stretch ratio of the chain at $t=+0$ (immediately after the deformation) and $f(t, \gamma)$ is a relaxation function reflecting the chain contraction process. We may assume that $h(\gamma)$ and $\alpha(\gamma)$ appearing in Eq. (7) are analytically expressed in a form deduced from the three-chain theory ${ }^{14}$

$$
\begin{aligned}
& h(\gamma)=\frac{6}{4+\gamma^{2}+\sqrt{4+\gamma^{2}}}, \\
& \alpha(\gamma)=\frac{1}{3} \operatorname{tr} \mathbf{C}^{-1 / 2}=\frac{1+\sqrt{4+\gamma^{2}}}{3} .
\end{aligned}
$$

Here, $\mathbf{C}^{-1 / 2}$ is the root mean square of Finger strain tensor, i.e., $\mathbf{C}^{-1}=\left(\mathbf{C}^{-1 / 2}\right)^{2}$. In fact, $h(\gamma)$ obtained from the simulation and experiments are excellently described by Eq. (8), as noted in Fig. 2 (compare solid curve and symbols). Thus, the essence of the nonlinear feature described by $h(t, \gamma)$ can be examined for the relaxation function $f(t, \gamma)$ appearing in Eq. (7).

This $f(t, \gamma)$ should relax through several modes. Specifically, we assume that $f(t, \gamma)$ relaxes through three modes as 


$$
f(t, \gamma)=\varphi_{1} \exp \left(-\frac{t}{\tau_{1}}\right)+\varphi_{2} \exp \left(-\frac{t}{\tau_{2}}\right)+\varphi_{3} \exp \left(-\frac{t}{\tau_{3}}\right)
$$

with the indices 1,2 , and 3 representing the fast, intermediate, and slow modes. The fractional intensities $\varphi_{i}$ change with $\gamma$ but satisfy a relationship $\varphi_{1}+\varphi_{2}+\varphi_{3}=1$ to fulfill a requirement of $f(t=+0, \gamma)=1$. Considering the linearity of the local force balance and the longitudinal Rouse processes, we set the fast and intermediate relaxation times to be independent of $\gamma$ and have known values, $\tau_{1}=\tau_{0}$ (unit time of simulation $=0.9 \mathrm{~s}$ and $\tau_{2}=\tau_{R}=\tau_{0}\langle Z\rangle_{\mathrm{eq}}{ }^{2} / 2 \pi^{2}$ (longest Rouse time in the PCN model $\left.{ }^{13}\right)=24 \mathrm{~s}$. Here $\tau_{R}$ is the Rouse time for stress relaxation in the PCN model. ${ }^{13}$ (Note that the relaxation time for the slowest Rouse mode and the rotational relaxation time of the Rouse chain are given by $2 \tau_{R}{ }^{2}$ ) Thus, utilizing $\tau_{3}$ and $\varphi_{i}$ as fitting parameters, we attempted to fit the $h(t, \gamma)$ data shown in Fig. 3 with Eqs. (7)-(10). A good fitting was achieved with the three-mode form of $f(t, \gamma)$ [Eq. (10)], and the best fit results, shown in Fig. 3 with the dashed curves, were obtained with a $\gamma$-independent $\tau_{3}$ $=230 \mathrm{~s}$ and $\gamma$-sensitive $\varphi_{i}$. This $\tau_{3}$ value is close to the value of the terminal relaxation time (reptation time) in the linear regime, $400 \mathrm{~s}$.

The $\gamma$-independence of $\tau_{3}$ naturally results in the timestrain separability at long $t>\tau_{3}$, and the change of $\varphi_{3}$ with $\gamma$ corresponds to the damping function $h(\gamma)$. Note, however, that the time-strain separability begins to hold approximately at shorter $t<\tau_{3}$ (at $t>\tau_{2}=\tau_{R}$ ) if the slow mode hardly contributes to $h(t, \gamma)$, i.e., if $\varphi_{3} \ll \varphi_{2}$ in a range of $\gamma$ of our focus. In relation to this point, we should note that the absolute magnitude of $\varphi_{3}$ is considerably smaller than that of $\varphi_{2}$; cf. $\left(\varphi_{2}, \varphi_{3}\right)=(0.62,0.17)$ for $\gamma=1.79$ (not very far from the linear viscoelastic regime $),\left(\varphi_{2}, \varphi_{3}\right)=(0.67,0.11)$ for $\gamma=2.56$, $\left(\varphi_{2}, \varphi_{3}\right)=(0.67,0.07) \quad$ for $\quad \gamma=3.07$, and $\left(\varphi_{2}, \varphi_{3}\right)$ $=(0.73,0.06)$ for $\gamma=4.02$ (well in the nonlinear regime). Thus, within an uncertainty of $\pm 10 \%$ and in particular for large $\gamma(>2.56)$, the slow mode contribution to $h(t, \gamma)$ is minor compared to the intermediate mode contribution and the time-strain separability holds even at $t \sim \tau_{2}\left(=\tau_{R}\right)$. This is the result reported earlier, ${ }^{4}$ which suggested that the time-strain separability being valid after completion of the longitudinal Rouse mode of the chain at $t \sim \tau_{R}{ }^{4}$ However, the slow mode contribution to $h(t, \gamma)$ is small but detectable, in particular for small $\gamma$, as already noted by Sanchez-Reyes and Archer ${ }^{5}$ and Inoue et al. ${ }^{6}$ Thus, rigorously speaking, the nonlinear damping occurs mainly through the longitudinal Rouse mode (intermediate mode) but also through the terminal, reptationlike mode (slow mode) at $t \sim \tau_{3}$ and the separability holds only after completion of this terminal mode.

\section{DISCUSSION}

The nonlinear damping under step shear strain has been attributed to the contraction of the chain along the array of entanglements. Thus, the finding of the previous studies ${ }^{5,6}$ confirmed in the previous section, rigorous validity of the time-strain separability only in the time scale of terminal relaxation $\tau_{3}$ (although the approximate validity within $\pm 10 \%$ is noted at shorter $t \sim \tau_{R}$ ), is a little puzzling because the contraction could be completed, in principle, through the Rouse motion. One possible origin of this retarded validity of the separability at $t>\tau_{3}$ is the tube pressure effect, as discussed by Mhetar and Archer. ${ }^{7}$ However, we may find another possible origin within the framework of the PCN simulation reported in this paper. In the remaining part of this paper, we first examine quantities that specify the chain contraction and then discuss the origin of the retarded validity of the time-strain separability.

\section{A. Quantities specifying the chain stretch/contraction}

The chain stretch/contraction can be quantified by the following relaxation functions.

For chain contour length $(L)$

$$
F_{\text {chain }}(t, \gamma)=K_{1}\left[\frac{\langle L(t)\rangle}{\langle L\rangle_{\mathrm{eq}}}-1\right] .
$$

For subchain length $(r)$

$$
F_{\text {subchain }}(t, \gamma)=K_{2}\left[\frac{\langle r(t)\rangle}{\langle r\rangle_{\mathrm{eq}}}-1\right] .
$$

For subchain stretch ratio defined with respect to quasiequilibrium reference

$$
\Lambda_{\text {subchain }}(t, \gamma)=K_{3}\left[\left\langle\frac{r(t)^{2}}{b^{2} n(t)}\right\rangle^{1 / 2}-1\right] .
$$

In Eqs. (11)-(13), \langle\rangle and \langle\rangle$_{\text {eq }}$ represent the ensemble average at a given time $t$ under strain $\gamma$ and at equilibrium, respectively: $\langle L\rangle_{\mathrm{eq}}=\langle r\rangle_{\mathrm{eq}}\langle Z\rangle_{\mathrm{eq}}$ and $\langle r\rangle_{\mathrm{eq}}=b\langle n\rangle_{\mathrm{eq}}^{1 / 2} \cdot K_{i}(i=1-3)$ is the normalization constant ensuring $F_{\text {chain }}(t=+0, \gamma)$ $=F_{\text {subchain }}(t=+0, \gamma)=\Lambda_{\text {subchain }}(t=+0, \gamma)=1$. Note that the average monomer number per subchain $\langle n(t)\rangle$ at time $t$ is different from the equilibrium number $\langle n\rangle_{\mathrm{eq}}$, and a quasiequilibrium size of the subchain composed of $\langle n(t)\rangle$ monomers defined with respect to this $\langle n(t)\rangle$ is given by $b\langle n(t)\rangle^{1 / 2}$. Thus, the function $\Lambda_{\text {subchain }}(t, \gamma)$ specifies the subchain stretch defined with respect to this quasi-equilibrium reference. In contrast, $F_{\text {chain }}(t, \gamma)$ and $F_{\text {subchain }}(t, \gamma)$ specify the ratios of the chain and subchain lengths to their real equilibrium lengths. Note also that the distributions of the subchain length and its stretch along the chain backbone are smeared in the averages $F_{\text {subchain }}(t, \gamma)$ and $\Lambda_{\text {subchain }}(t, \gamma)$ taken for all subchains.

Utilizing the simulated values of $r(t)$ and $n(t)$ at each time $t$ during the stress relaxation process, we evaluated the relaxation functions defined above. In Fig. 4, these functions are plotted against the reduced time $t / \tau_{R}$ (symbols). The dashed curves denote the DE prediction (cf. Appendix). The terminal relaxation times of $F_{\text {chain }}(t, \gamma), F_{\text {subchain }}(t, \gamma)$, and $\Lambda_{\text {subchain }}(t, \gamma)$ are insensitive to $\gamma$, and the time evolution of the chain and subchain lengths is well characterized by these functions.

As noted in Fig. 4(a), the chain length recovers its equilibrium value (i.e., $F_{\text {chain }}(t, \gamma)$ decays to zero) via three steps. The fast step occurring at $t \leq \tau_{0}$ results from the local force 

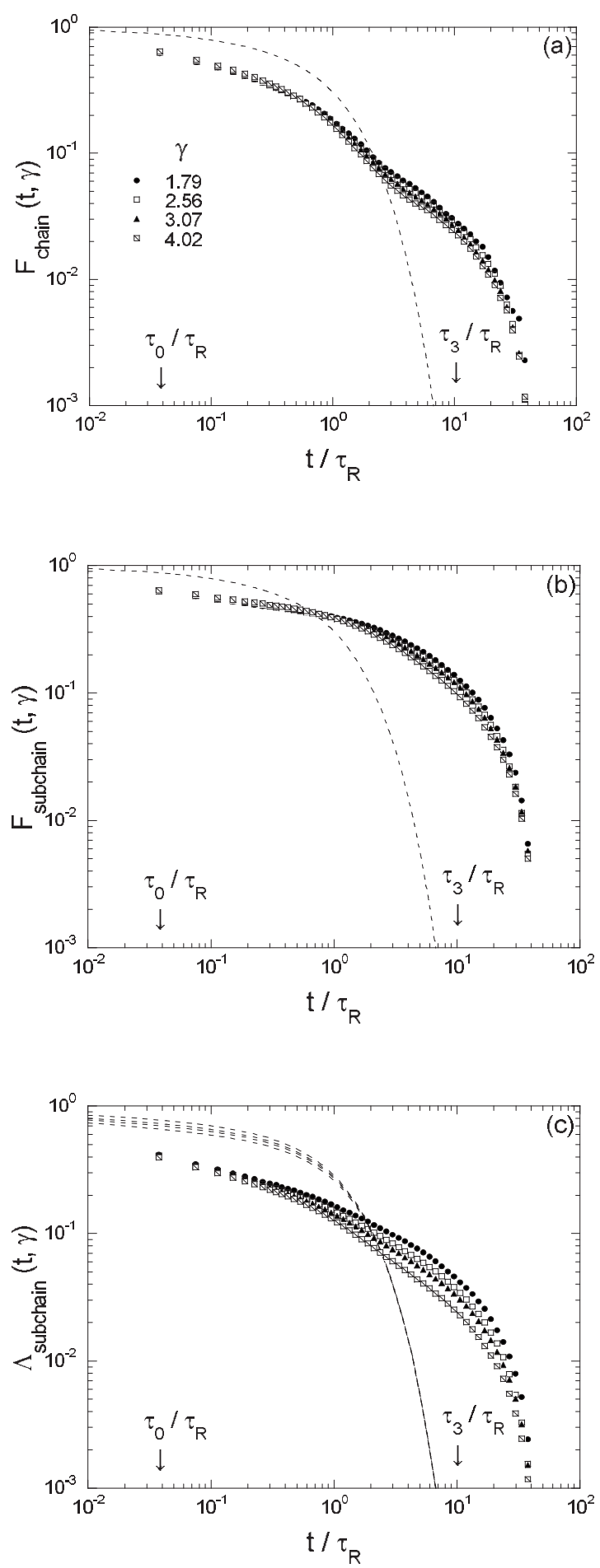

FIG. 4. Relaxation of (a) contour length of the chain, (b) subchain length, and (c) subchain stretch defined with respect to the quasiequilibrium reference (based on the monomer number in the subchain at respective times) Dashed lines indicate the prediction of DE theory (see Appendix).

balance process which is similar, in a sense, to the process $\mathrm{X}$ proposed by $\operatorname{Lin}^{22}$ within the framework of the fixed tube model. The intermediate process occurring at $t \sim \tau_{R}$ is due to the intrinsic, longitudinal Rouse motion of the chain as considered in the DE model. The recovery of the equilibrium chain length completes up to $\sim 90 \%$ through this intrinsic Rouse process, but the recovery of the remaining $10 \%$ requires the slow process occurring in a time scale of $\tau_{3}$ de-

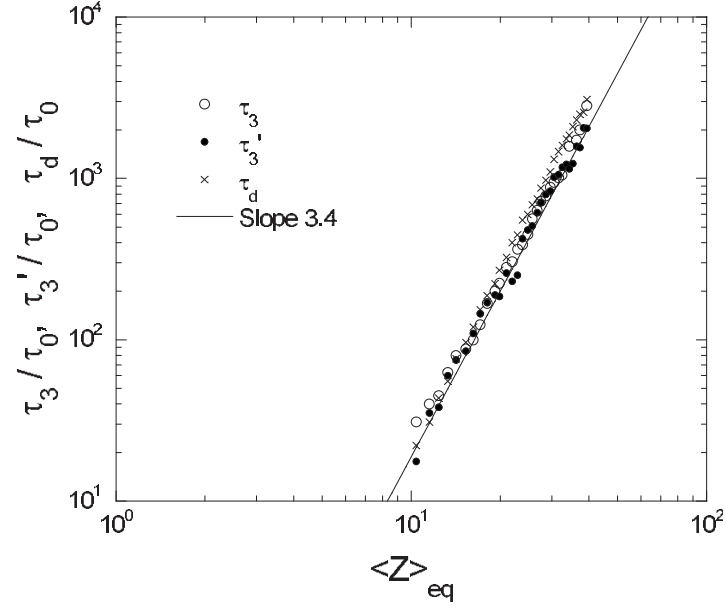

FIG. 5. Chain length dependence of the relaxation time $\tau_{3}^{\prime}$ extracted from the subchain length relaxation function. For comparison, the characteristic time $\tau_{3}$ of the slow mode defined for the time-dependent damping function and the terminal relaxation time $\tau_{d}$ in the linear viscoelastic regime are also shown.

fined for the time-dependent damping function (Fig. 3). Thus, the retarded chain contraction, corresponding to the retarded validity of the time-strain separability, is directly confirmed for $F_{\text {chain }}(t, \gamma)$.

In contrast to this behavior of the chain length, the relaxation of $F_{\text {subchain }}(t, \gamma)$ and $\Lambda_{\text {subchain }}(t, \gamma)$ proceeds almost in one-step and completes at $t \sim \tau_{3}$; cf. Figs. 4(b) and 4(c). Thus, the dominant part of the length equilibration is slower for the subchain than for the whole contour of the chain; compare $F_{\text {chain }}(t, \gamma)$ and $F_{\text {subchain }}(t, \gamma)$. This difference is discussed later in relation to the number of entanglements per chain and the tension distribution along the chain backbone.

For specifying the dominant mechanism for the slow equilibration of the subchain length, we conducted the simulations for the chains of various lengths and evaluated $F_{\text {subchain }}(t, \gamma)$. The results were very similar to those seen in Fig. 4(b) (and not shown here), and we were able to evaluate the terminal relaxation time $\tau_{3}{ }^{\prime}$ defined for $F_{\text {subchain }}(t, \gamma)$. In Fig. 5, this $\tau_{3}{ }^{\prime}$ is reduced by the unit time of simulation $\tau_{0}$ and plotted against the equilibrium entanglement number per chain, $\langle Z\rangle_{\mathrm{eq}}\left(\propto\right.$ total monomer number per chain, $\left.n_{\mathrm{tot}}\right)$. For comparison, the characteristic time $\tau_{3}$ of the slow mode defined for $f(t, \gamma)$ involved in the time-dependent damping function [cf. Eq. (10)] and the terminal relaxation time $\tau_{d}$ in the linear viscoelastic regime are also shown. These characteristic times are close to each other and proportional to $\langle Z\rangle_{\mathrm{eq}}{ }^{3.4}$. This result confirms that the reptative motion of the chain dominates the recovery of the subchain length [as well as the onset of the rigorous time-strain separability of $G(t, \gamma)]$.

\section{B. Origin of slow length equilibration}

Now, we examine the origin of the slow equilibration of the subchain length seen in Fig. 4(b). At $t=t_{R}$, the chain length has been almost equilibrated $\left(\left\langle L\left(\tau_{R}\right)\right\rangle \cong 1.1\langle L\rangle_{\mathrm{eq}}\right.$ for $\gamma \cong 3$ ) but the subchain length equilibration is retarded to $t$ $\sim \tau_{d}$; cf. Figs. 4(b) and 4(c). This retardation is intimately related to the time-evolution of the sliplink number $Z$ per 

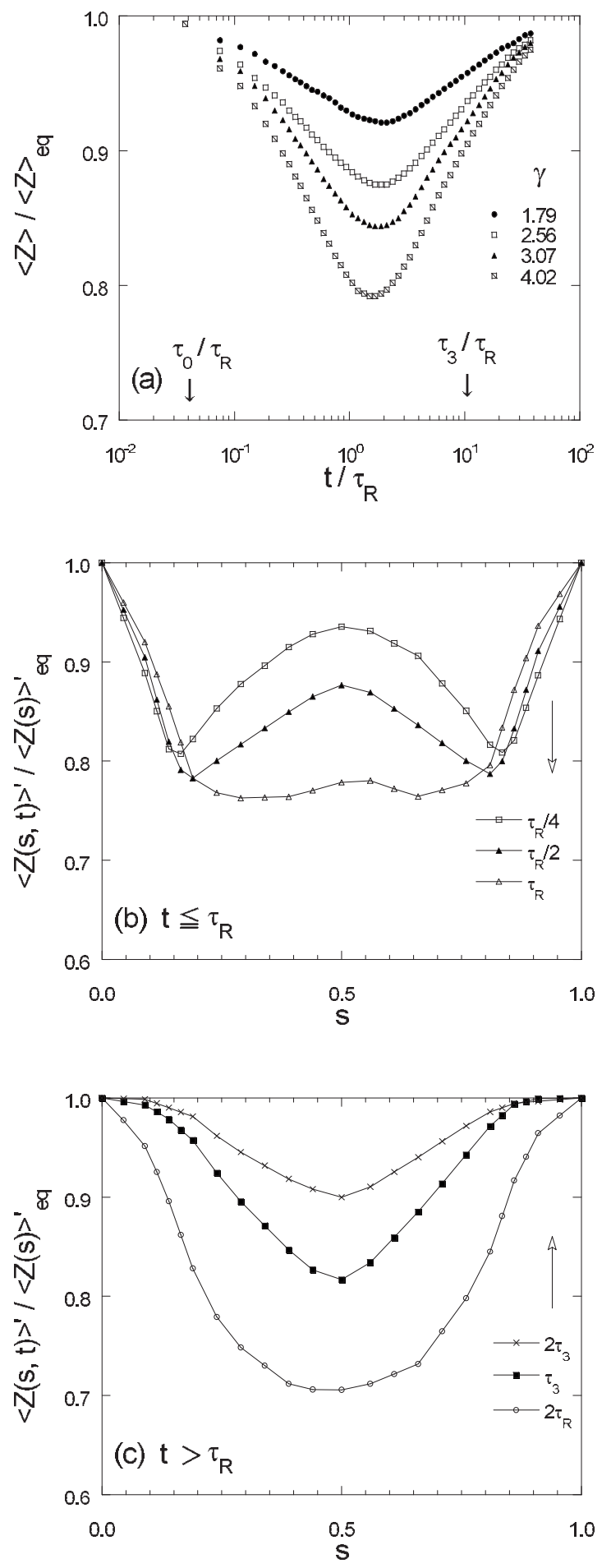

FIG. 6. Time-evolution of the sliplink number under step strain: Average total sliplink number per chain for various strain (part a) and curvilinear sliplink density at $\gamma=4.02$ for $t \leq \tau_{R}$ (part b) and $t>\tau_{R}$ (part c). Arrows in parts (b) and (c) indicate the direction of time evolution.

chain and the sliplink density along the chain. In Fig. 6(a), the total sliplink number per chain $\langle Z(t)\rangle=n_{\text {tot }}\langle\langle n(t)\rangle$ at respective $t$ is reduced by its equilibrium value, $\langle Z\rangle_{\text {eq }}$, and plotted against the reduced time $t / \tau_{R}$. Figures 6(b) and 6(c) show similarly reduced plots for the sliplink density $\langle Z(s, t)\rangle^{\prime}$ at a reduced curvilinear coordinate $s=x /\langle L(t)\rangle$, where $x$ is the curvilinear distance of the sliplink measured from one end of the chain and \langle\rangle$^{\prime}$ indicates an average taken for all subchains at the given coordinate $s$. The $\langle Z(t)\rangle /\langle Z\rangle_{\text {eq }}$ ratio decreases with increasing $t$ up to $2 \tau_{R}$. This decrease is mainly attributable to the CCR effect, i.e., the removal of sliplinks for a given chain due to the contraction of surrounding chains that occurs through the intrinsic, longitudinal Rouse mode. Detailed features of this process are noted for $\langle Z(s, t)\rangle^{\prime}$ : The sliplink density first decreases at portions near chains ends (at $s \sim 0.1$ and 0.9 ), and this decrease propagates to the center portion $(s \sim 0.5)$ at intermediate $t \sim \tau_{R}$. Finally, the equilibrium sliplink density is recovered at the center and end portions, with the recovery being faster for the latter.

In relation to the slow recovery of the total number and density of the sliplink seen above, we here focus on the average length of the subchain $\left\langle r\left(\tau_{R}\right)\right\rangle$ at the time $\tau_{R}$

$$
\begin{aligned}
&\left\langle r\left(\tau_{R}\right)\right\rangle \cong \frac{\left\langle L\left(\tau_{R}\right)\right\rangle}{\left\langle Z\left(\tau_{R}\right)\right\rangle}=\frac{\left\langle L\left(\tau_{R}\right)\right\rangle}{\langle L\rangle_{\mathrm{eq}}} \times \frac{\langle Z\rangle_{\mathrm{eq}}}{\left\langle Z\left(\tau_{R}\right)\right\rangle} \times\langle r\rangle_{\mathrm{eq}} \\
& \text { with }\langle r\rangle_{\mathrm{eq}}=\frac{\langle L\rangle_{\mathrm{eq}}}{\langle Z\rangle_{\mathrm{eq}}}
\end{aligned}
$$

Thus, $\langle r(t)\rangle$ remains considerably larger than $\langle r\rangle_{\mathrm{eq}}$ [by a factor of 1.4 for $\gamma \cong 3$; cf. Fig. 4(b)] even at $t \sim \tau_{R}$ where the intrinsic, Rouse contraction is completed and the chain length has been almost equilibrated $\left(\left\langle L\left(\tau_{R}\right)\right\rangle \cong 1.1\langle L\rangle_{\mathrm{eq}}\right.$ for $\gamma \cong 3)$. In other words, the CCR-induced increase of $\langle n(t)\rangle$ (decrease of $\langle Z(t)\rangle)$ is one of the factors that lead to the retarded equilibration of the subchain length. The full equilibration of the subchain length requires full reformation of the sliplinks (recovery of $\langle Z\rangle_{\mathrm{eq}}$ and $\langle Z(s)\rangle_{\mathrm{eq}}{ }^{\prime}$ ) which occurs through the large-scale reptative motion in the time scale of $\tau_{3}{ }^{\prime} \cong \tau_{d}$, as noted for $F_{\text {subchain }}(t, \gamma)$ [Fig. 4(b)].

Concerning the above argument, we should also note that the subchain stretch $\Lambda_{\text {subchain }}(t, \gamma)$ has only moderately relaxed at $t \sim \tau_{R}$ where $\langle n(t)\rangle$ begins to decrease $(\langle Z(t)\rangle$ begins to increase); compare Figs. 4(c) and 6(a). Namely, the increase of $\langle n(t)\rangle$ is not the only factor that retards the subchain length equilibration. The other factor resulting in this retardation can be found in relation to the subchain tension, as discussed below.

As can be noted from Fig. 6(a), the average monomer number per subchain, $\langle n(t)\rangle \cong\langle n\rangle_{\mathrm{eq}}\langle Z\rangle_{\mathrm{eq}}\langle Z(t)\rangle$, is larger than $\langle n\rangle_{\text {eq }}$ throughout the stress relaxation process. Thus, the average spring constant of the subchain, $\langle\kappa(t)\rangle \propto 1 /\langle n(t)\rangle$, is smaller than that at equilibrium and the subchain tension, the driving force of the chain contraction, is weaker than that expected for the intrinsic Rouse contraction considered in the DE model. This weak tension leads to the retarded equilibration of the chain/subchain length. For more quantitative argument, we should note a fact that the chain contracts because the tension is larger at around the chain center than at the chain ends. In other words, the retarded equilibration reflects a distribution of the subchain tension along the chain backbone. The tension at the curvilinear coordinate $s$ and time $t$ is given by 

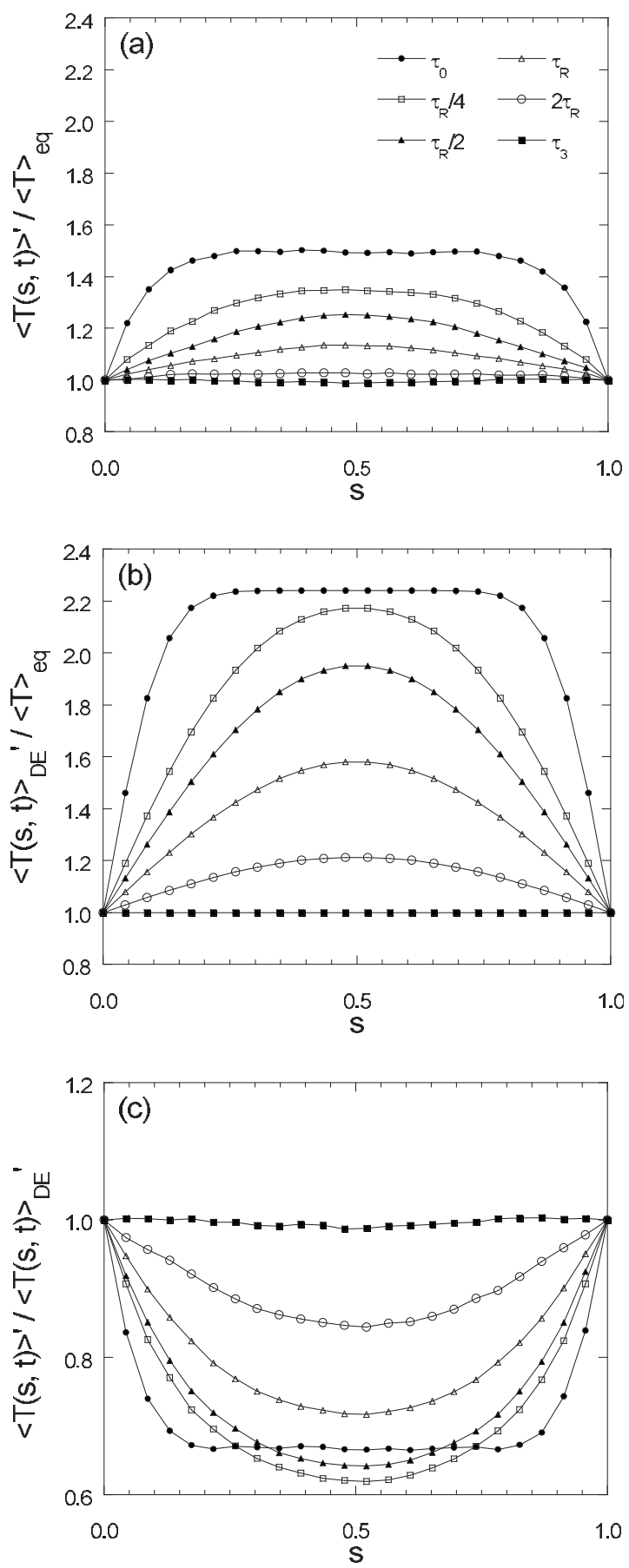

FIG. 7. Curvilinear distribution of normalized subchain tension under step strain $\gamma=4.02$ obtained from (a) simulation in this study and (b) DE model. Part (c) shows a ratio of the stress obtained from the simulation to that deduced from the DE model.

$$
\langle T(s, t)\rangle^{\prime} \cong\left\langle\frac{r(s, t)}{n(s, t)}\right\rangle^{\prime} .
$$

In Fig. $7(\mathrm{a}),\langle T(s, t)\rangle^{\prime}$ is normalized by the equilibrium tension $\langle T\rangle_{\mathrm{eq}}$. For comparison, $\langle T(s, t)\rangle_{\mathrm{DE}}{ }^{\prime}$ deduced from the DE model (for the Rouse contraction along the fixed tube axis; cf. Appendix) is shown in Fig. 7(b). For both of our PCN simulation and DE model, the tension is larger at around the chain center than at the chain ends to activate the chain contraction. More importantly, this tension gradient along the chain backbone is less significant for our simulation through- out the stress relaxation process. In other words, the tension at around the chain center is smaller in our simulation than in the DE model in particular at $t \leq \tau_{R}$, as most clearly seen for the $\langle T(s, t)\rangle^{\prime} /\langle T(s, t)\rangle_{\mathrm{DE}}{ }^{\prime}$ ratio [Fig. $\left.7(\mathrm{c})\right]$. The weak tension at around the chain center is due to a reduction of the spring constant (a rise of the monomer number per segment) in this section of the chain, corresponding to the minimum of the sliplink density shown in Figs. 6(b) and 6(c). This reduction of the tension at around the chain center is the dominant factor that results in the retarded equilibration of the subchain length in our simulation.

In relation to this point, we should note that the relaxation of the subchain stretch $\Lambda_{\text {subchain }}(t, \gamma)$ is slower in our simulation than in the DE model; cf. Fig. 4(c). Enhancement of the chain tension due to this retarded relaxation of $\Lambda_{\text {subchain }}(t, \gamma)$ is overwhelmed by the softening of the subchain (decrease of the spring constant) due to the CCRinduced increase of $\langle n(t)\rangle$ (cf. Fig. 6) thereby giving a net decrease of the chain tension at around the chain center to retard the subchain length equilibration.

Concerning this result for the subchain length equilibration, we should again point out that the dominant part of the chain length equlibration (contraction) is faster than the subchain length equilibration and occurs at $t \sim \tau_{R}$. A cancellation between the increase of subchain length $\langle r(t)\rangle$ (due to the increase of $\langle n(t)\rangle$ and the subchain stretch) and the decrease of $\langle Z(t)\rangle$, both due to CCR, allows the chain length $\langle L(t)\rangle$ $=\langle r(t)\rangle\langle Z(t)\rangle$ to be almost equilibrated at $t \sim \tau_{R}$, although the subchains have not been equilibrated at such short $t$. Furthermore, the softening of the subchains (due to the increase of $\langle n(t)\rangle)$ tends to help the completion of the nonlinear damping of the stress, which results in the approximate validity (within $\pm 10 \%$ ) of the time-strain separability at $t \ll \tau_{d}$ (at $t$ $\sim \tau_{R}$ ). At the same time, we should emphasize that the delicate failure of this separability at $t \sim \tau_{R}$ reflects the nonlinear dynamics of the chain under step shear (not the simple Rouse contraction along the fixed tube), as discussed earlier.

\section{Comment for the tube pressure effect}

Our PCN simulation, incorporating no tube, led us to attribute the retarded equilibration of the chain/subchain length to the CCR-induced decrease of $\langle Z(t)\rangle$ (increase of $\langle n(t)\rangle)$ and the corresponding reduction of the tension gradient (distribution) along the chain backbone. Within the context of the tube model, this mechanism of retarded equilibration appears to correspond to CCR-induced tube dilation that occurs predominantly at around the chain center [see Figs. 6(b) and 6(c)]. In contrast, Mhetar and Archer ${ }^{7}$ attributed the retarded equilibration of the chain length to the tube pressure effect, the lateral squeezing from the strained tube having a uniform diameter smaller than that at equilibrium. Thus, at this moment, these two views do not appear to match with each other. It is an important subject of future work to experimentally test these views (through scattering experiments for partially labeled chains, for example) and further examine if the matching of those views can be achieved within the generalized tube model that explicitly incorporates the heterogeneity of the tube diameter along the chain backbone. 


\section{CONCLUSION}

Using the PCN simulations, we investigated the nonlinear stress damping and the chain contraction under step shear in sliplink network. The simulation reproduced a delicate failure of the time-strain separability of the nonlinear relaxation modulus $G(t, \gamma)$ experimentally observed at $t \sim \tau_{R}$ (longest Rouse relaxation time). For this delicate failure, the chain contraction was examined through relaxation functions defined for the whole contour length of the chain, the subchain length between sliplinks, and the subchain stretch based on the monomer number of the subchain at respective times. These functions commonly exhibited the relaxation occurring through three modes, the fast mode due to local force balance around entanglement occurring at $t \sim \tau_{0}$ (unit time of the simulation), the intermediate Rouse contraction mode occurring at $t \sim \tau_{R}$, and the slow mode due to reptative network rearrangement occurring at $t \sim \tau_{d}$. The relaxation function for the chain length was dominated by the intermediate Rouse mode, although the slow reptative mode had a non-negligible contribution to retard the terminal relaxation up to $t \sim \tau_{d}$. In contrast, the relaxation functions for the subchain length and stretch were largely contributed from the slow reptative mode. Thus, the chain/subchain length equilibration was completed at $t \sim \tau_{d}$, which resulted in the delicate failure of the time-strain separability seen for $G(t, \gamma)$ at $t \sim \tau_{R}$. This retarded equilibration was related to the CCRactivated decrease of the entanglement number per chain, to the corresponding softening of the subchain (due to the increase of the monomer number per chain), and most importantly to the reduction of the subchain tension at around the chain center compared to the DE prediction.

A comment needs to be made for the sliplink removal/ reformation algorithm. The PCN simulation, being based on the same algorithm as used in the present study, did not reproduce experimental results for the large-scale conformational relaxation under moderately fast steady shear flow, the shear-rate insensitivity of the end-to-end vector fluctuation. ${ }^{23-25}$ Thus, the reduction of sliplink number due to steady flow during the time scale of this relaxation $\left(\sim \tau_{d}\right)$ appeared to be less significant than assumed in the model algorithm, and a mechanism of enhancing the entanglement reformation, the hidden entanglement appearance (affecting the slow dynamics in the time scale of $\tau_{d}$ ), was incorporated in the simulation. ${ }^{23,24}$ However, it remains uncertain if this mechanism works in the transient state during the nonlinear stress relaxation process occurring basically at $t<\tau_{d}$. A test for this point is considered to be an interesting/ important subject in our future work.

We would also emphasize again that the simulation presented in this paper, made for moderately entangled chains $\left(\langle Z\rangle_{\mathrm{eq}} \sim 20\right)$ in a microscopic cell, just tested the assumption of the independence of the contraction and orientation relaxation and did not resolve the shear banding/yielding observed experimentally for highly entangled chains. ${ }^{15-17}$ Nevertheless, the shear banding/yielding could be examined through a simulation for highly entangled chains in a macroscopic cell. This examination is considered to be another important subject of our future work.

\section{APPENDIX: 1D ROUSE MOTION IN DE THEORY}

In the 1D Rouse model proposed in Ref. 2, the curvilinear coordinate of the $n$th segment $s_{n}(t)$ at time $t$ is written as

$$
s_{n}(t)=Y_{0}(t)+2 \sum_{p=1}^{\infty} Y_{p}(t) \cos \left(\frac{p \pi n}{N}\right)+\frac{\bar{L}}{N} n,
$$

where $\bar{L}$ is equilibrium value of contour length. $Y_{p}(t)$ is normal mode amplitude defined by

$$
\begin{aligned}
Y_{0}(t) \equiv & \frac{1}{N} \int_{0}^{N} d n s_{n}(t) \\
Y_{p}(t) \equiv & \frac{1}{N} \int_{0}^{N} d n \cos \left(\frac{p \pi n}{N}\right)\left(s_{n}(t)-\frac{\bar{L}}{N} n\right) \\
& \text { for } \quad p=1,2, \cdots .
\end{aligned}
$$

The first and second moments of the normal modes after step strain are provided by Wen and Hua ${ }^{26}$

$$
\begin{aligned}
& \left\langle Y_{p}(t)\right\rangle=-\frac{2(\alpha-1)}{p^{2} \pi^{2}} \bar{L} \exp \left(-\frac{p^{2} t}{\tau_{R}}\right) \text { for odd } p, \\
& \left\langle Y_{p}(t)\right\rangle=0 \text { for even } p \\
& \left\langle Y_{p}(t)^{2}\right\rangle=\frac{N(\alpha-1)^{2}}{2 p^{2} \pi^{2}} \bar{L}^{2} \exp \left(-\frac{2 p^{2} t}{\tau_{R}}\right),
\end{aligned}
$$

where $\alpha \equiv\langle|\mathbf{E} \cdot \mathbf{u}|\rangle_{\mathrm{eq}}$ with $\mathbf{E}=$ deformation gradient tensor and $\mathbf{u}=$ unit vector.

From Eqs. (A1) and (A4), the mean contour length of chain can be written as

$$
\begin{aligned}
\langle L(t)\rangle & =\left\langle s_{N}(t)-s_{0}(t)\right\rangle=\bar{L}-4 \sum_{p \text { :odd }}^{\infty}\left\langle Y_{p}(t)\right\rangle \\
& =\bar{L}\left[1+(\alpha-1) \sum_{p \text { :odd }}^{\infty} \frac{8}{p^{2} \pi^{2}} \exp \left(-\frac{p^{2} t}{\tau_{R}}\right)\right] .
\end{aligned}
$$

Thus, relaxation function for the whole contour length $F_{\text {chain }}(t, \gamma)$ defined by Eq. (11) is given by

$$
F_{\text {chain }}^{\mathrm{DE}}(t)=\frac{\langle L(t)\rangle-\bar{L}}{\langle L(t=+0)\rangle-\bar{L}}=\sum_{p \text { : odd }}^{\infty} \frac{8}{p^{2} \pi^{2}} \exp \left(-\frac{p^{2} t}{\tau_{R}}\right),
$$

In the DE model, the average subchain length is given by $\langle r(t)\rangle=\langle L(t)\rangle / N$ and thus the relaxation function for the subchain length $F_{\text {subchain }}(t, \gamma)$ defined by Eq. (12) is identical to $F_{\text {chain }}^{\mathrm{DE}}(t)$.

The mean-square subchain length is expressed as 


$$
\begin{aligned}
\left\langle r(t)^{2}\right\rangle= & \frac{1}{N} \sum_{n=1}^{N}\left\langle\left(s_{n+1}(t)-s_{n}(t)\right)^{2}\right\rangle=\frac{1}{N} \int_{0}^{N} d n\left\langle\left(\frac{\partial s_{n}}{\partial n}\right)^{2}\right\rangle \\
= & \frac{1}{N} \sum_{p=1}^{N} \frac{2 p^{2} \pi^{2}}{N}\left\langle Y_{p}(t)^{2}\right\rangle-\frac{8}{N} \frac{\bar{L}}{N_{p}} \sum_{p \text { odd }}^{\infty}\left\langle Y_{p}(t)\right\rangle+\left(\frac{\bar{L}}{N}\right)^{2} \\
= & \left(\frac{\bar{L}}{N}\right)^{2}\left[1+(\alpha-1)^{2} \frac{1}{N} \sum_{p=1}^{N} \exp \left(-\frac{2 p^{2} t}{\tau_{R}}\right)\right. \\
& \left.+2(\alpha-1) \sum_{p \text { :odd }}^{\infty} \frac{8}{p^{2} \pi^{2}} \exp \left(-\frac{p^{2} t}{\tau_{R}}\right)\right] .
\end{aligned}
$$

Thus, the subchain stretch ratio defined by Eq. (13) is readily obtained as

$$
\begin{aligned}
\Lambda_{\text {subchain }}^{\mathrm{DE}}(t, \gamma)= & \frac{1}{\alpha-1}\left[\left\{1+(\alpha-1)^{2} \frac{1}{N} \sum_{p=1}^{N} \exp \left(-\frac{2 p^{2} t}{\tau_{R}}\right)\right.\right. \\
& \left.\left.+2(\alpha-1) \sum_{p \text { :odd }}^{\infty} \frac{8}{p^{2} \pi^{2}} \exp \left(-\frac{p^{2} t}{\tau_{R}}\right)\right\}^{1 / 2}-1\right] .
\end{aligned}
$$

Finally, the tension at the curvilinear coordinate $s=n / N$ can be calculated as

$$
\begin{aligned}
\langle T(s, t)\rangle_{\mathrm{DE}}^{\prime}= & \frac{3 k_{B} T}{b^{2}}\left\langle\frac{\partial s_{n}(t)}{\partial n}\right\rangle \\
= & \frac{3 k_{B} T}{b^{2}}\left[-2 \sum_{p=1}^{\infty}\left\langle Y_{p}\right\rangle \frac{p \pi}{N} \sin \left(\frac{p \pi n}{N}\right)+\frac{\bar{L}}{N}\right] \\
= & \langle T\rangle_{\mathrm{eq}}\left[1+4(\alpha-1) \sum_{p \text { odd }}^{\infty} \frac{1}{p \pi} \sin (p \pi s)\right. \\
& \left.\times \exp \left(-\frac{p^{2} t}{\tau_{R}}\right)\right] .
\end{aligned}
$$

Here, $\langle T\rangle_{\mathrm{eq}}=\left(3 k_{B} T / b^{2}\right)(\bar{L} / N)=3 k_{B} T / a$ (=independent of $\left.s\right)$.

${ }^{1}$ P.-G. de Gennes, Scaling Concepts in Polymer Physics (Cornell University Press, Ithaca, 1980).

${ }^{2}$ M. Doi and S. F. Edwards, The Theory of Polymer Dynamics (Clarendon, Oxford, 1986).

${ }^{3}$ K. Osaki, S. Kimura, and M. Kurata, J. Polym. Sci., Polym. Phys. Ed. 19, 517 (1981).

${ }^{4}$ K. Osaki, K. Nishizawa, and M. Kurata, Macromolecules 15, 1068 (1982).

5 J. Sanchez-Reyes and L. A. Archer, Macromolecules 35, 5194 (2002).

${ }^{6}$ T. Inoue, Y. Yamashita, and K. Osaki, Macromolecules 35, 1770 (2002).

${ }^{7}$ V. Mhetar and L. A. Archer, J. Non-Newtonian Fluid Mech. 81, 71 (1999).

${ }^{8}$ G. Marrucci and B. de Cindio, Rheol. Acta 19, 68 (1980).

${ }^{9}$ G. Marrucci, J. Non-Newtonian Fluid Mech. 62, 279 (1996).

${ }^{10}$ C. Y. Chen, S. M. Wu, Z. R. Chen, T. J. Huang, and C. C. Hua, J. Polym. Sci., Part B: Polym. Phys. 41, 1281 (2003).

${ }^{11}$ C. C. Hua and J. D. Schieber, J. Chem. Phys. 109, 10018 (1998).

${ }^{12}$ Y. Masubuchi, J. I. Takimoto, K. Koyama, G. Ianniruberto, G. Marrucci, and F. Greco, J. Chem. Phys. 115, 4387 (2001).

${ }^{13}$ K. Furuichi, C. Nonomura, Y. Masubuchi, H. Watanabe, G. Ianniruberto, F. Greco, and G. Marrucci, Rheol. Acta 47, 591 (2008).

${ }^{14}$ G. Marrucci, F. Greco, and G. Ianniruberto, J. Rheol. 44, 845 (2000).

${ }^{15}$ S. Q. Wang, S. Ravindranath, P. Boukany, M. Olechnowicz, R. P. Quirk, A. Halasa, and J. Mays, Phys. Rev. Lett. 97, 187801 (2006).

${ }^{16}$ S. Ravindranath and S. Q. Wang, Macromolecules 40, 8031 (2007).

${ }^{17}$ P. E. Boukany, S.-Q. Wang, and X. Wang, Macromolecules 42, 6261 (2009).

${ }^{18}$ Y. Masubuchi, G. Ianniruberto, F. Greco, and G. Marrucci, J. NonNewtonian Fluid Mech. 149, 87 (2008).

${ }^{19}$ K. Furuichi, C. Nonomura, Y. Masubuchi, G. Ianniruberto, F. Greco, and G. Marrucci, J. Soc. Rheol., Jpn. 35, 73 (2007).

${ }^{20}$ M. Takahashi, T. Isaki, T. Takigawa, and T. Masuda, J. Rheol. 37, 827 (1993).

${ }^{21}$ K. Osaki, H. Watanabe, and T. Inoue, Macromolecules 29, 3611 (1996).

${ }^{22}$ Y. H. Lin, Macromolecules 17, 2846 (1984).

${ }^{23}$ Y. Masubuchi, H. Watanabe, G. Ianniruberto, F. Greco, and G. Marrucci, J. Soc. Rheol., Jpn. 32, 197 (2004).

${ }^{24}$ Y. Masubuchi, K. Furuichi, K. Horio, T. Uneyama, H. Watanabe, G. Ianniruberto, F. Greco, and G. Marrucci, J. Chem. Phys. 131, 114906 (2009).

${ }^{25}$ T. Uneyama, Y. Masubuchi, K. Horio, Y. Matsumiya, H. Watanabe, J. A. Pathak, and M. C. Roland, J. Polym. Sci., Part B: Polym. Phys. 47, 1039 (2009).

${ }^{26}$ Y. H. Wen and C. C. Hua, J. Polym. Sci., Part B: Polym. Phys. 44, 1199 (2006). 